Radi al Transport Char acteri stics of Fast I ons Due to Energeti c- Parti cl e Modes i nsi de the Last $\mathrm{Cl}$ osed- $\mathrm{Fl}$ ux Surface in the Compact Hel i cal Syst em

\begin{tabular}{|l|l|}
\hline $\begin{array}{l}\text { jour nal or } \\
\text { publ i cat i on ti t l e }\end{array}$ & Physi cal Revi ew Let ter s \\
\hline vol une & Vol . 100 \\
\hline page range & 065005- 1 - 065005-4 \\
\hline year & 2008- 02-01 \\
\hline URL & ht t p: //hdl . handl e. net /10655/3900 \\
\hline
\end{tabular}




\title{
Radial Transport Characteristics of Fast Ions Due to Energetic-Particle Modes inside the Last Closed-Flux Surface in the Compact Helical System
}

\author{
Kenichi Nagaoka, ${ }^{1}$ Mitsutaka Isobe, ${ }^{1}$ Kazuo Toi, ${ }^{1}$ Akihiro Shimizu, ${ }^{1}$ Akihide Fujisawa, ${ }^{1}$ Shunsuke Ohshima, ${ }^{1}$ \\ Haruhisa Nakano, ${ }^{1}$ Masaki Osakabe, ${ }^{1}$ Yasushi Todo, ${ }^{1}$ Tsuyoshi Akiyama, ${ }^{1}$ Yoshihiko Nagashima, ${ }^{2}$ Chihiro Suzuki, ${ }^{1}$ \\ Shin Nishimura, ${ }^{1}$ Yasuo Yoshimura, ${ }^{1}$ Keisuke Matsuoka, ${ }^{1}$ and Shoichi Okamura ${ }^{1}$ \\ ${ }^{1}$ National Institute for Fusion Science, 322-6 Oroshi, Toki 509-5292, Japan \\ ${ }^{2}$ Kyushu University, Kasuga 816-8580, Japan \\ (Received 3 October 2007; published 15 February 2008)
}

\begin{abstract}
The internal behavior of fast ions interacting with magnetohydrodynamic bursts excited by energetic ions has been experimentally investigated in the compact helical system. The resonant convective oscillation of fast ions was identified inside the last closed-flux surface during an energetic-particle mode (EPM) burst. The phase difference between the fast-ion oscillation and the EPM, indicating the coupling strength between them, remains a certain value during the EPM burst and drives an anomalous transport of fast ions.
\end{abstract}

DOI: 10.1103/PhysRevLett.100.065005

Fast-ion-confinement is one of the most important issues for future burning plasma experiments, such as ITER, since a dominant heating process in such plasmas is an alpha particle heating produced by deuterium-tritium nuclear reactions. In particular, interaction between fast ions and magnetohydrodynamics (MHD) modes is a key issue for fast-ion-confinement [1]. Fast ions may excite various MHD modes, and resulting anomalous transport of the fast ions occurs due to the interaction with the MHD activities [2-7].

In many fusion devices, various Alfvén eigenmodes (AEs) have been destabilized by fast ions in frequency ranges absent of shear Alfvén continuum damping [8]. The characteristics of AEs depend on the plasma parameters and agree well with experimental observations. On the other hand, energetic-particle modes (EPMs) are determined by characteristics of the fast-ion motions such as transit, bounce and precession, and can be destabilized with the frequencies inside the shear Alfvén continuum when the energetic-ion drive is strong enough to overcome the continuum damping [9]. Actually, EPMs are experimentally observed on the condition that the fast-ion pressure is fairly high [3]. EPMs often show nonlinear phenomena such as periodic burst and frequency chirping, which are considered to be related to fast-ion behavior, for example, change of fast-ion profile. The investigation of EPM properties and interaction with fast ions is still in progress $[3,10,11]$.

Recently, a directional Langmuir probe (DLP) method was applied to plasmas heated by a neutral beam (NB) for fast-ion measurement, and fast-ion behavior inside the last closed-flux surface (LCFS) can be measured with high time and spatial resolutions [12]. In this Letter, we focus on the experimental studies on the fast-ion-behavior interacting with bursting EPMs. The interaction characteristics between fast ions and the EPM and anomalous transport of fast ions due to EPM are discussed.
PACS numbers: 52.55.Pi, 52.35.Bj, 52.35.Py, 52.55.Hc

The stage of our experiments is a helical device (stellarator), named compact helical system (CHS) [13]. The target plasma is heated and sustained by tangential NB injection with port-through power of $800 \mathrm{~kW}$, the beam duration of $100 \mathrm{~ms}$ and beam energy of $40 \mathrm{keV}$. The plasma parameters are magnetic field strength $B=0.9 \mathrm{~T}$, lineaveraged electron density $n_{e}=0.5-1.0 \times 10^{19} \mathrm{~m}^{-3}$, central electron temperature $T_{e}(0) \sim 0.3 \mathrm{keV}$, normalized plasma pressure $\beta_{p} \sim 0.1 \%$. When the normalized beam pressure increased to similar values as the plasma pressure, fast-ion-driven MHD bursts with the poloidal and toroidal mode numbers of $m=3 / n=2$ were observed periodically. The frequency of the bursts chirps down and is lower than the Alfvén gap frequency $\left(f_{\text {gap }} \sim\right.$ $170 \mathrm{kHz}$ for toroidicity-induced AE with $n=1$ ). The burst mode rotates in the ion-diamagnetic direction. From these experimental observations, this mode was identified as EPM [14,15]. Anomalous loss of energetic ions due to EPM burst was observed by a neutral particle analyzer (NPA) [16] and a loss fast-ion probe (LIP) [17]. The EPM bursts enhanced loss of transit ions with the energy of the injected NB [15].

A DLP was installed in CHS and a two dimensional probe drive system controls the DLP position in $R-z$ plane. The schematic of the DLP position and the magnetic surface mainly utilized in this experiment are shown in Fig. 1(a). The DLP has seven Langmuir probe channels: four face to the codirected flux (ch1, 3, 5, 7) and three to the counter-directed flux (ch2, 4, 8) [see Fig. 1(b)]. A Mirnov coil was also installed on the DLP (ch6) to measure the magnetic fluctuation $\partial B_{\theta} / \partial t$ at the same point with fast-ion measurement. The flux of codirected transit fast ions can not reach the probe channels facing to counterdirected flux, because the gyro-radius of fast ions is the same order to the DLP diameter, and it can be given by $I_{\text {fast ion }}=I_{\text {co }}-I_{\text {counter }}[12,18]$. The experimental demonstration of fast-ion measurement using the DLP was per- 


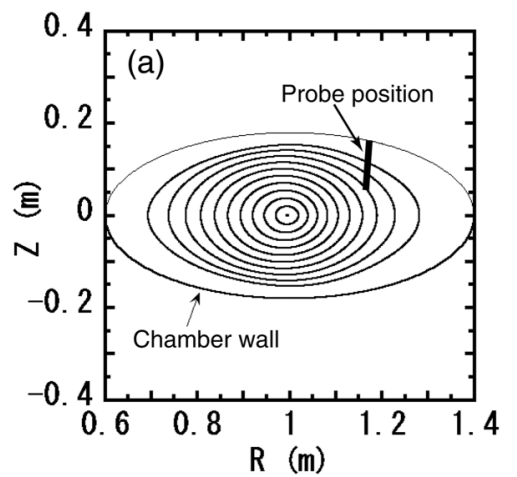

(b)

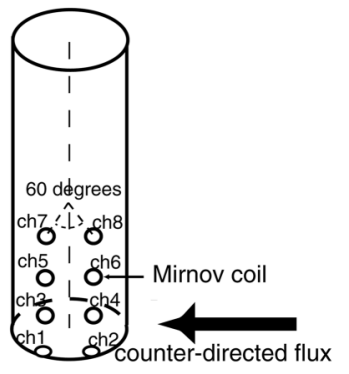

FIG. 1. The schematics of (a) the magnetic flux surface in a horizontally elongated cross section in CHS with the magnetic axis of $R_{\mathrm{ax}}=97.4 \mathrm{~cm}$, and the position of DLP and (b) the DLP channel arrangement.

formed in CHS and a good agreement with NPA and LIP observations was obtained [12].

The simultaneous measurements of the fast ions and the magnetic fluctuation were performed inside of the LCFS using the DLP. The wave forms of the magnetic fluctuation of an EPM burst, codirected and counterdirected ion fluxes are shown in Fig. 2(a). The codirected ion flux increases during the EPM burst while the counterdirected flux remains unchanged. The increase of codirected flux with the EPM is recognized as a fast-ion flux enhanced by the EPM burst [17]. The frequency of the EPM chirps down from $120 \mathrm{kHz}$ to $70 \mathrm{kHz}$ [see Fig. 2(b)], and the codirected (fastion) flux enhanced by the EPM burst shows significant coherence to the EPM in comparison with counterdirected (bulk ion) flux [see Figs. 2(c) and 2(d)]. As seen from Fig. 2(a), the fast-ion flux enhanced by the EPM consists of two parts: one is a fast part oscillating with the EPM, and the other is a slow part, having triangular shape and slow time scale of $\sim 0.5 \mathrm{msec}$, which is shown by the dotted red line in the figure.

Figure 3 shows the simultaneous measurement of fastion-responses to the EPM in different minor radii at $r / a^{*}=0.91,0.96$ and 1.01, where $r$ and $a^{*}$ are minor radius and averaged plasma radius with the vacuum configuration, respectively. The peak of slow part of fast-ion response propagates in the outward direction with the velocity of $600 \mathrm{~m} / \mathrm{s}$. The time scale of fast-ion loss from the center toward outside of the plasma is $\sim 0.5 \mathrm{msec}$ and corresponds to the burst duration of the EPM, which implies that the pulse duration of the burst can be determined by the time scale of the fast-ion transport.

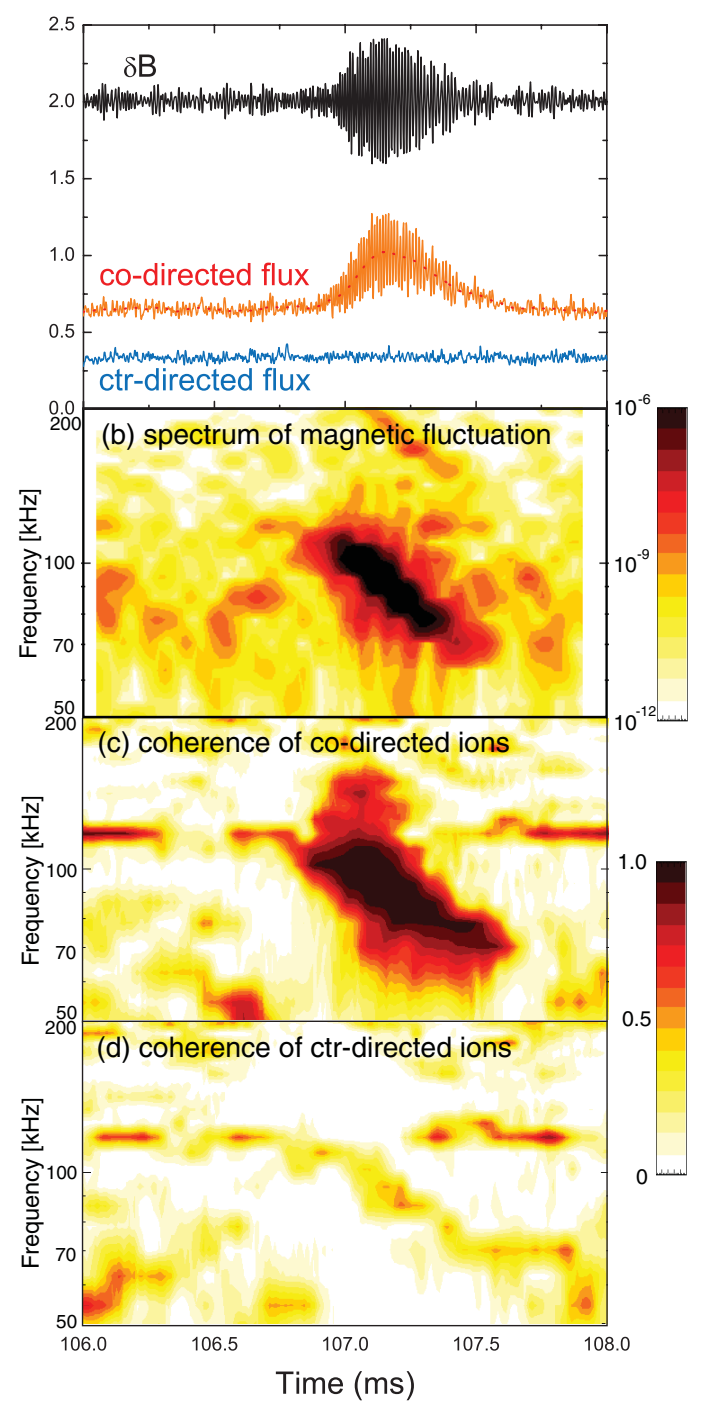

FIG. 2 (color). (a) The wave forms of magnetic fluctuation (black line), co- (red line) and counterdirected (blue line) ion fluxes at $r / a^{*}=0.975$. The dotted red line shows the slow part of the fast-ion response. (b) The power spectrum of the EPM. The coherences between the fast-ion response (codirected flux) and the EPM (c), and between the bulk-ion response (counterdirected flux) and the EPM (d).

The mode profile of the EPM near the LCFS was measured by the DLP, which is shown in Fig. 4(a). The LCFS is located at $r / a^{*} \sim 1.05$ in this experiment due to the plasma and fast-ion pressure effects. The peak of eigenfunction of the EPM is located around $r / a^{*}=0.7$, and the eigenfunction terminates at the LCFS as seen in Fig. 4(a). The profiles of the fast-ion responses to the EPM bursts were also measured by the DLP and are shown in Figs. 4(b) and 4(c). The fast part of fast-ion-response locates inside the LCFS, while the slow part was observed not only inside but also outside of the LCFS. These results are consistent with the previous observations of fast-ion-loss due to EPMs. The LIP located outside of the LCFS observed the slow part of the fast-ion response and no fast responses. The slow part more strongly decays radially than the depen- 


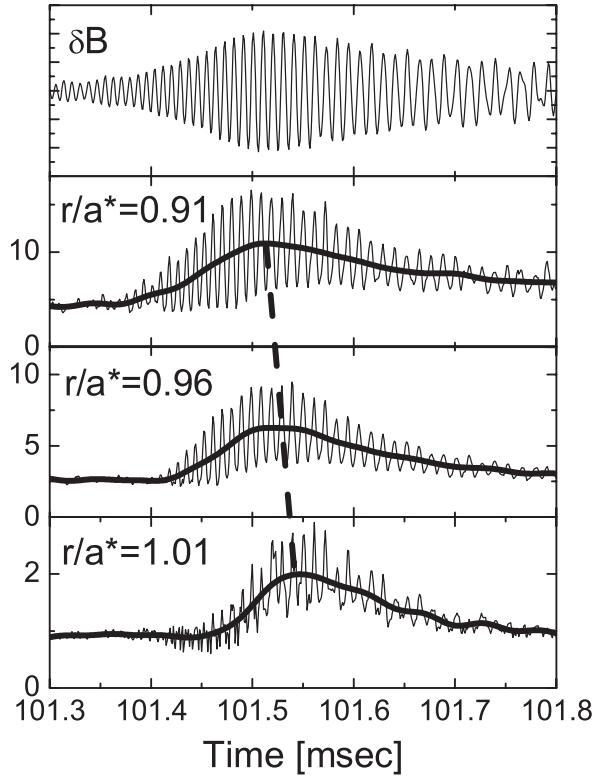

FIG. 3. The wave forms of magnetic field of an EPM and fastion responses observed at $r / a^{*}=0.91,0.96$, and 1.01 .

dence of $r^{-1}$ [dotted line in Fig. 4(c)], indicating the slow part includes redistribution component of fast ions.

The fast and slow parts of the fast-ion response to the EPM increase with the amplitudes of the EPM at the position, which is shown in Fig. 5. The fast part has a linear relation with the EPM amplitude, indicating that the fast part is a convective response of the fast ions. It is noted that the vertical axis of Fig. 5(a) is not a lost ion flux, so it is a different point from the convective loss of fast ions discussed in Ref. [16]. According to incompressibility of the EPM, the fast part is considered to be proportional to the density gradient of fast ions:

$$
\delta \Gamma_{\text {fast response }} \propto\left|\operatorname{grad} n_{f}\right| \delta B .
$$

On the other hand, the slow part is proportional to the square of the EPM amplitude, which is discussed later.

The weak coherence of the bulk ion (counterdirected) flux with the EPM can be seen in Fig. 2(d), indicating the bulk ions also oscillate with the EPM. The phase relations of fast- and bulk-ion response to the EPM are shown in Fig. 6, in which the vertical axis is the phase of fast- and bulk-ion flux to the Mirnov signal of $\partial B_{\theta} / \partial t$ observed at the same position. The bulk ions oscillate in phase $\left(\phi_{b}=\right.$ 0 ) with the EPM, meaning that the bulk ions has same phase with radial component of the magnetic fluctuation, $\delta B_{r}$. Thus the bulk ions keep a frozen-in condition during the EPM burst. On the other hand, the phase relation of the fast-ion response is significantly different from frozen-in condition, and keeps a certain phase $\left(\phi_{f} \neq 0\right)$. This is clear evidence that the interaction of fast ions with the EPM is different with that of the bulk ions. It is noted that the coupling strength between fast ions and the EPM remains unchanged during the EPM.

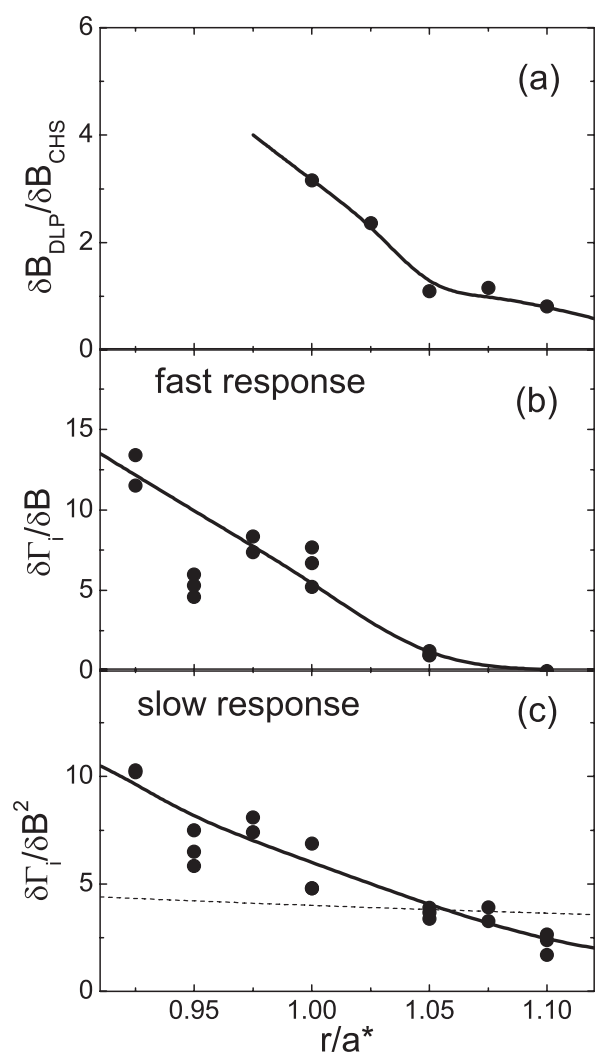

FIG. 4. The profiles of (a) mode amplitude of the EPMs, (b) the fast part and (c) the slow part of fast-ions responses. The dotted line shows the dependence of $r^{-1}$.

Here we discuss about radial flux of fast ions induced by the EPM, which is given by

$$
\Gamma_{r} \sim\left\langle\delta n_{f}\left[\delta V_{r}+V_{\|}\left(\delta B_{r} / B\right)\right]\right\rangle,
$$

where $\delta n_{f}, \delta V_{r}, V_{\|}, \delta B_{r}, B$ and $\langle A\rangle$ are amplitude of fastion-density fluctuation, radial component of oscillating velocity induced by electric field of the $\operatorname{EPM}(\delta \mathbf{E})$, velocity of fast ions parallel to the magnetic field, radial component of magnetic field of the EPM, the magnetic field strength and integral over a period of the EPM, respectively. The density fluctuation of fast ions, $\delta n_{f}$, has a certain phase with the EPM ( $\delta V_{r}$ and $\left.\delta B_{r}\right)$; thus, the integral in Eq. (2) does not vanish and statistically irreversible transport occurs. When the density fluctuation of fast ions, $\delta n_{f}$, is maximum value at the probe position, both the radial velocity $\left(\delta V_{r}\right)$ and radial component of the EPM field $\left(\delta B_{r}\right)$ estimated by the magnetic fluctuation measured at the same position are in the outward direction, and they are in the inward when $\delta n_{f}$ is minimum. Therefore the averaged radial flux induced by the EPM is in the outward direction. From Eq. (1), the fast-ion flux induced by the EPM is proportional to the second power of the EPM amplitude: $\Gamma_{r} \propto\left|\operatorname{grad} n_{f}\right| \delta B^{2}$. It is concluded that the slow part of fastion response to the EPM can be understood by the radial component of fast ions flux given by Eq. (2). 


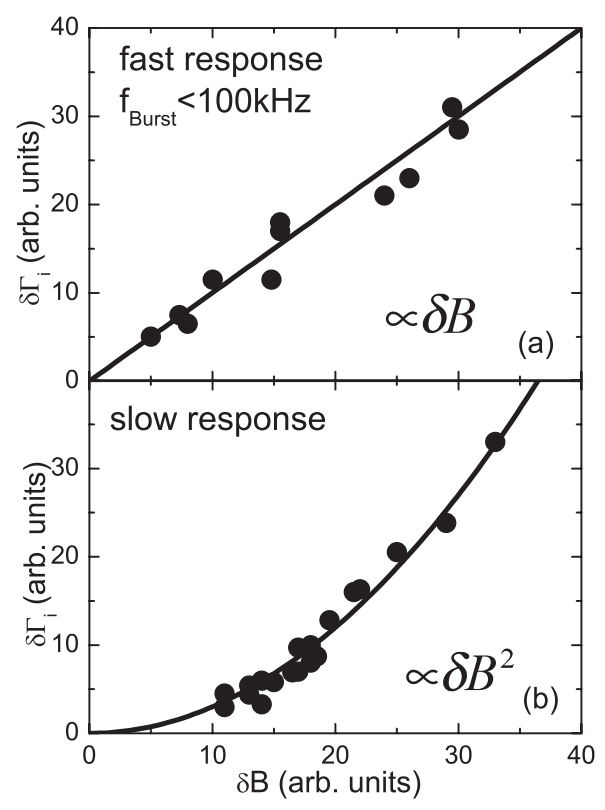

FIG. 5. The fast-ion fluxes of (a) fast and (b) slow parts as a function of the EPM amplitude.

The drive force of the EPM is given by $F_{D} \propto\left\langle\delta n_{f} \boldsymbol{V}_{D}\right.$. $\delta \boldsymbol{E}\rangle$, where $\boldsymbol{V}_{D}$ is drift velocity of fast ions [19]. At the probe position, the drift velocity is dominated by grad- $B$ drift and its direction is in the ion-diamagnetic direction. In the similar way with Eq. (2), the finite phase produces the irreversible drive force, and the EPM is destabilized by the fast ions $\left(F_{D}>0\right)$ at the probe position. In order to evaluate the total growth rate of the EPM, the integration of $F_{D}$ over the plasma volume is necessary, which is not so simple, because the direction of drift velocity changes in the poloidal direction with helical component of $m=2$. The orbits of codirected transit ions shift outward; thus, the total growth rate is considered to be $F_{D}>0$. From Eq. (1), the dependence of growth rate of the EPM is obtained as $\gamma \propto F_{D} / \delta B^{2} \propto\left|\operatorname{grad} n_{f}\right|$, which is consistent with theoretical prediction $[9,20]$.

The direct observation of fast-ion behaviors using the DLP method was performed, and the two kinds of fast-ion responses, fast and slow parts, were observed inside the LCFS. The fast part is resonant convective response oscillating with the EPM. The observed phase relation of fastion oscillation to the EPM indicates the strong coupling between them, which keeps a constant during frequency chirping, and produces statistically irreversible transport of fast ions. Thus, the slow part is identified as the anomalous transport induced by the EPM, including both losing and redistributing components. The dependence of growth rate is consistent with theoretical prediction. An unstable EPM is predicted in the reversed shear case of ITER [21]. Once EPMs are excited in burning plasmas, the modification of the fast-ion profile is considered to be inevitable due to the strong link between anomalous transport of fast ions and the drive of the EPMs. And the suppression of the EPM

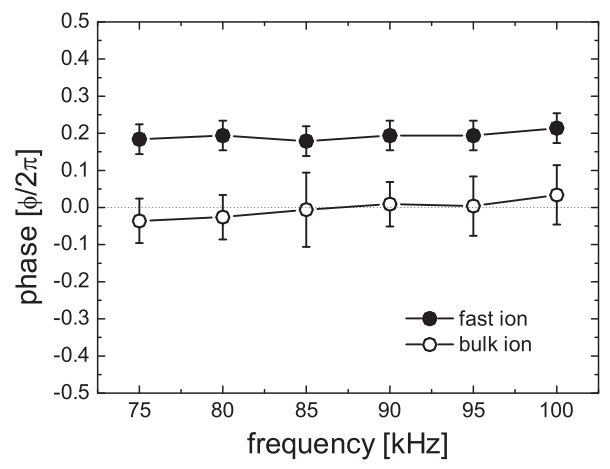

FIG. 6. The phase relations at $r / a^{*}=0.975$ between the fast ions (codirected ion flux) and $\partial B_{\theta} / \partial t$ (closed circles), and between the bulk plasma (counterdirected ion flux) and $\partial B_{\theta} / \partial t$ (open circles).

amplitude as well as the stabilization of the EPM is significantly important because of the linear dependence of fast-ion flux on the squared amplitude of the EPM.

One of the authors (K. N.) would like to thank Professors K. Itoh (NIFS), W. W. Heidbrink (UC Irvin) and Dr. K. Shinohara (JAEA) for their fruitful discussions. This work was supported by NIFS (No. NIFS05ULPD609), partially by a Grand-in-Aid of the Ministry of Education, Culture, Sports, Science and Technology of Japan (No. 15740333) and by the Sumitomo Foundation (No. 030943).

[1] W. W. Heidbrink et al., Phys. Fluids B 5, 2176 (1993).

[2] W. W. Heidbrink et al., Nucl. Fusion 34, 535 (1994).

[3] E. D. Fredrickson et al., Phys. Plasmas 13, 056109 (2006).

[4] Y. Kusama et al., Nucl. Fusion 39, 1837 (1999).

[5] A. Weller et al., Phys. Rev. Lett. 72, 1220 (1994).

[6] K. Toi et al., Nucl. Fusion 40, 1349 (2000).

[7] M. Osakabe et al., Proc. 29th Eur. Conf. on Control. Fusion and Plasma Phys. (2002) PI-083.

[8] C. Z. Chen and M.S. Chance, Phys. Fluids 29, 3695 (1986).

[9] L. Chen, Phys. Plasmas 1, 1519 (1994).

[10] F. Zonca et al., Phys. Plasmas 9, 4939 (2002).

[11] Y. Todo, Phys. Plasmas 13, 082503 (2006).

[12] K. Nagaoka et al., Plasma Fusion Res. 1, 005 (2006).

[13] K. Matsuoka et al., Proc. 12th Int. Conf. on Plasma Physics and Controlled Nuclear Fusion Research 1988: Proceedings of the 12th IAEA Conference Nice, 1988 (IAEA, Vienna, 1988) Vol. 2, p. 411.

[14] K. Toi et al., J. Plasma and Fusion Res. Series 5, 50 (2002).

[15] M. Isobe et al., Nucl. Fusion 46, S918 (2006).

[16] H. Matsushita et al., Rev. Sci. Instrum. 75, 3607 (2004).

[17] M. Isobe et al., Rev. Sci. Instrum. 70, 827 (1999).

[18] K. Nagaoka et al., J. Plasma and Fusion Res. Series (to be published).

[19] M. N. Rosenbluth and P. H. Rutherford, Phys. Rev. Lett. 34, 1428 (1975).

[20] C.Z. Cheng, N. N. Gorelenkov, and C. T. Hsu, Nucl. Fusion 35, 1639 (1995).

[21] A. Fasoli et al., Nucl. Fusion 47, S264 (2007). 